\title{
Comparison of resolution specifications for micro- and nanometer measurement techniques
}

\author{
Weckenmann/Albert, Tan/Özgür, Shaw/Laura, Zschiegner/Nils \\ Chair Quality Management and Manufacturing Metrology (QFM) \\ University Erlangen-Nuremberg \\ Naegelsbachstrasse 25 D-91052 Erlangen Germany \\ Tel: +49 91318526540 Fax: +49 91318526524 \\ qfm@qfm.uni-erlangen.de
}

In most of the measurement tasks of micro- and nanotechnology, a high resolution of the instrument is required. However in most cases, resolution specification of the manufacturer could not be verified due to the lack of an acceptance test for the experimental investigations. In many cases, specification of the resolution is directly taken from the manufacturing specifications, which are mostly theoretically calculated values and do not always represent the real performance of the system. Instrument specific influencing factors, like the noise of data or the effect of optics are also needed to be considered during the investigation of real resolution performance. Because of this, it is required not only to develop calibration and testing tools but also to apply specific acceptance tests. In this article, results of the application of a Siemens-Star to investigate lateral resolution of different instruments are presented. Different surface measurement techniques such as white light interferometer, atomic force microscope and chromatic sensor are used as measurement devices. Results are compared not only with each other but also with manufacturer specifications. By this, the application of calibration and testing equipments for the investigation of resolution specifications is demonstrated.

Keywords: Micro- and Nanotechnology, resolution, surface measurement, practice-oriented

\section{Introduction}

Advances in micro- and nanotechnology show that, without understanding the product details and describing them quantitatively, it is not possible to develop them further. Measurements of such products give the required information [1]. One of the important requirements while choosing a measurement device is the suitability of its resolution. But the problem to choose a right resolution is the lack of information about the specification of the resolution. The fundamental definition has been given in DIN 1319-1 as "smallest detectable difference between two indications on a displaying device" [2]. Since this is a general definition, there are different ways to calculate it. In some cases, resolution specifications depend on very theoretical methods; such as Sparrow or Rayleigh criteria or in other cases, such as dividing the field of a view by the number of pixels does not represent the real resolution performance. Real resolution capacity depends on other factors like quality of optics, noise of data and etc. Another important restriction in optical measurements, which also has to be taken into account, is the dependency of lateral resolution power on the wavelength of the light and the numerical aperture of the lens. Although this definition of diffraction limited resolution has been mostly used in community of microscopy, it is not an accepted definition in most other fields. It could be said that there is not any agreed way to specify lateral resolution for the areal measurement techniques.

In this study we have presented an application of a concept from macro techniques in the field of microand nanometrology. Different types of Siemens-Star structures are investigated with different measurement instruments.

\section{Testing Tool: Siemens-Star}

In classical image processing applications, Siemens-Stars which have equal number of dark and white structures (mostly 32) are used to investigate the resolution of cameras. These structures have an alternating width in radial direction. They seem like they touch each other, but the only place where they really intersect is the centre of star (see figure 1). Application of these structures has been modified and applied to the investigation of lateral resolution of measurement devices in micro- and nanotechnologies. The dark and white colours, which are shown in figure 1, are substituted with grooves which have a constant depth value. The circumference of this ambiguous region, which can be seen in the middle of 
structure, gives information about the resolution performance of the instrument. The equation to calculate the number of the resolution is given as the following.

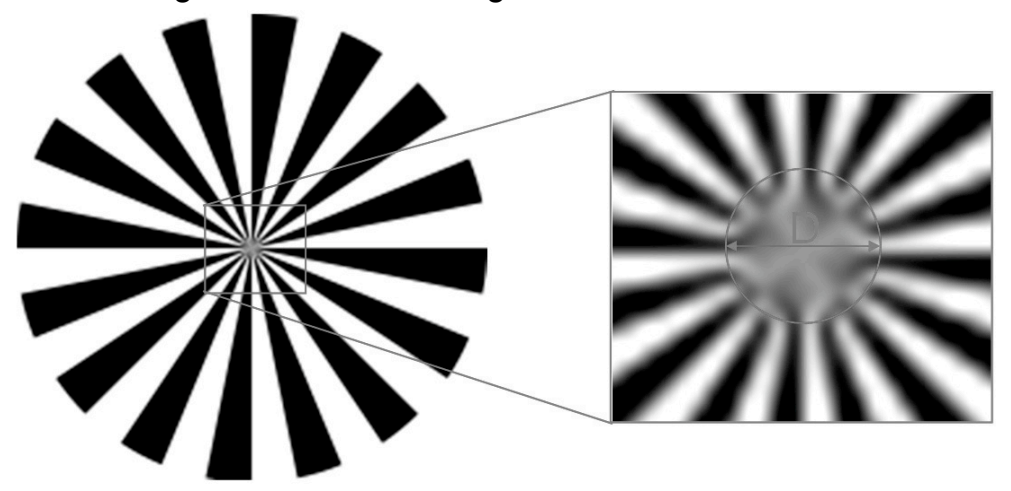

Figure 1: A Siemens-Star for the evaluation of resolution of optical instruments

If the total number of these structures is $n$ and the diameter of this ambiguous region is $D$, lateral resolution can be calculated as follow:

$$
\text { Lateral Resolution }=\frac{\pi \times D}{n}
$$

Technique of Focus Ion Beam (FIB) is applied to make the structures on the surface. Two different types of stars are investigated. In the first part of the measurements, 5 different stars with a diameter of $60 \mu \mathrm{m}$ and a step height of $200 \mathrm{~nm}$ are measured. In the second part a star with a diameter of $100 \mu \mathrm{m}$ and the same step height has been investigated.

This idea has been used not only in measurement of camera resolutions [3] but also during the investigation of new measurement techniques, like [4].

\section{Measurement Principles}

In order to compare the lateral resolution of different measurement principles, techniques of white light interferometry, chromatic aberration and atomic force microscopy are investigated.

White light interferometer (WLI): Due to its high vertical resolution, WLI has been accepted as an important tool for surface topography measurements. A beam splitter separates the light coming from the source into two beams. One of the beams reaches to the object and the other to the reference (see figure 2). The Mireau objective is driven by a linear actuator element along the optical axis. During the movement in vertical direction, the intensity of the reflected light is stored for each pixel in the CCD element. The measurement region depends on the working distance of the actuator. The maximum of intensity modulation in the interference correlogram occurs at a position where the distance to the measuring object is equal to the distance to the reference surface. This maximum is evaluated to get the height data of the test sample at a certain point. Finally, the height data together with the corresponding lateral coordinates give the topography of the surface.
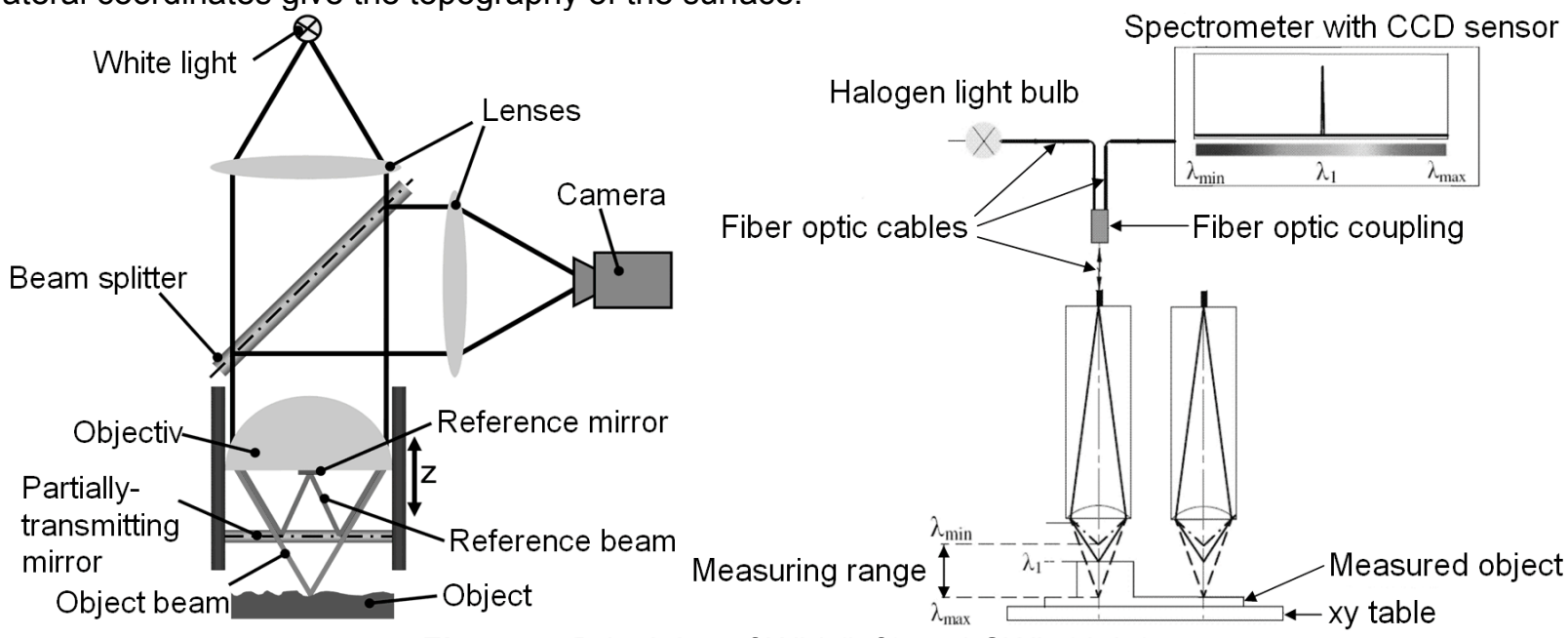

Figure 2: Principles of WLI (left) and CWL (right) 
In this work a state-of-the-art white light interferometer (Taylor Hobson $\mathrm{CCl} 1000$ ) with three different objectives has been used to measure the Siemens-Stars. It has a vertical resolution of $0.01 \mathrm{~nm}$ with a vertical working range of $400 \mu \mathrm{m}$. The main disadvantage of used WLI compared to AFM measurements is that, being an optical technique, the lateral resolution is limited to around $0.35 \mu \mathrm{m}$.

Chromatic White Light Sensor. The measurement principle of the second sensor is based on the chromatic aberration of white light. The refractive index of the front lens in the sensor head changes for different wavelengths of light. As the focus length of the lens depends on the refractive index, a lens with a strong chromatic aberration will show the focus point of the different wavelengths at different positions along the optical axis (see figure 2). This effect, the longitudinal chromatic aberration, is the measurement principle applied in the chromatic sensor. White light, which is passed through a passive lens, is separated into different coloured focal points and focused on the sample. The intensity of the reflected light is evaluated with a spectrometer. As the wavelength which was focused on the sample surface has the maximum intensity, the distance between the sensor and the sample surface can be determined on the basis of comparison tables. The measuring range in $z$-axis is equal to the distance from blue and red focus point, of $300 \mu \mathrm{m}$. The vertical resolution over the $z$ range of the sensor is $10 \mathrm{~nm}$. With the chromatic sensor surface structures up to $2 \mu \mathrm{m}$ (effective spot diameter of the white light) can be resolved.

Atomic Force Microscope (AFM): In general it can be said that AFMs are primarily designed to measure surface topography with a very high spatial resolution. A fine tip at the end of a cantilever for the investigation of the sample surface, a feedback sensor which detects small changes of the tip or cantilever position, a z scanner which keeps the probe under constant conditions (i.e. force) and a xy scanner to displace the tip are the main components of AFMs.

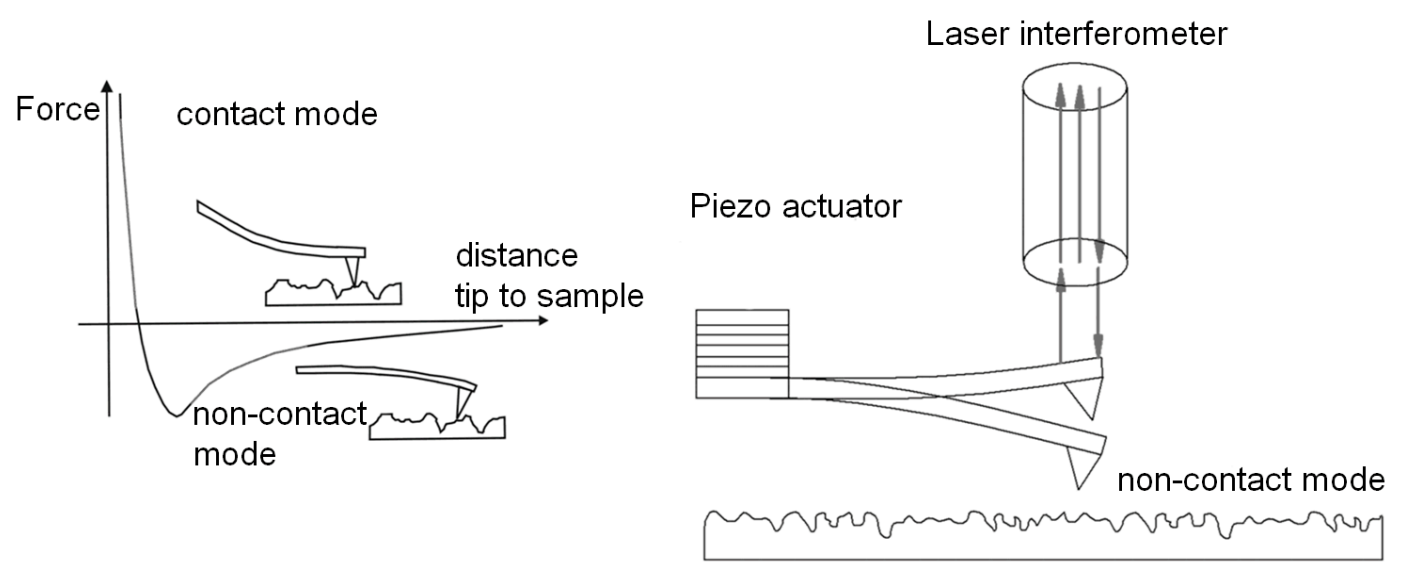

Figure 3: Measuring Principle of Atomic Force Microscope

The cantilever with a sharp tip, mounted on the end of the piezo scanning tube, is moved towards the surface and when it is very close (a few tens of nanometers) the surface forces result in an interaction between the surface and the tip. The main modes for operating an AFM are contact and non-contact. In the contact mode the cantilever is scanned across the surface and repulsive surface forces cause a bending of the cantilever. In the dynamic non-contact mode the cantilever is oscillated close to its resonant frequency above the surface. As seen in figure 3 , to detect the position of the cantilever, light from a laser diode is, through a fiber-optic cable, led to the sensor head. The light reflects from two planes, the planar end of the fibre (reference beam) and the upper side of the cantilever (object beam). The resulting interference signal is detected by a photodiode at the end of the optical fibre. When the distance between the cantilever and the optical fibre is changing, the resulting change in interference signal can be used as the input to a feedback system that ensures the force between the sample and tip (and hence distance) is kept constant. Restrictions in z-measurement range and the needed measurement time could be seen as the main disadvantages. The AFM, which is used in this work, allows a scanning range in the xy axis of $80 \times 80 \mu \mathrm{m}$ with a lateral resolution of $5 \mathrm{~nm}$. In the z-axis the measuring range is limited to $6 \mu \mathrm{m}$. 


\section{Measurements with Siemens-Star}

\subsection{Experiments with WLI}

Three different objectives have been used for this study. As seen in table 1, not only the theoretical values of objectives are given (dividing field of view by the number of pixels), but also the physical limits to resolution namely, numerical apertures are shown. Additionally optical resolution of the systems, which are calculated with Sparrow criteria $(\lambda=570 \cdot \mathrm{nm})$, are listed.

Table 1: Overview of objectives that have applied for the investigations

\begin{tabular}{cccc}
\hline Objectives & $\begin{array}{c}\text { Lateral Resolution in } \\
\boldsymbol{\mu m}\end{array}$ & $\begin{array}{c}\text { Numerical } \\
\text { Aperture }\end{array}$ & $\begin{array}{c}\text { Opt. lateral resolution in } \\
\boldsymbol{\mu m}\end{array}$ \\
\hline $10 \mathrm{X}$ & 1.76 & 0.3 & 0.95 \\
$20 \mathrm{X}$ & 0.88 & 0.4 & 0.71 \\
$50 \mathrm{X}$ & 0.35 & 0.55 & 0.52 \\
\hline
\end{tabular}

In figure 4, measurement of star Nr. 1 with three different objectives can be seen. Before the presentation of any further results, it is clear that, as the resolution decreases, size of the ambiguous region increases.

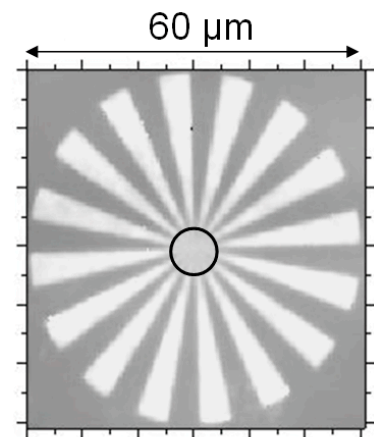

a) $50 \times$ objective

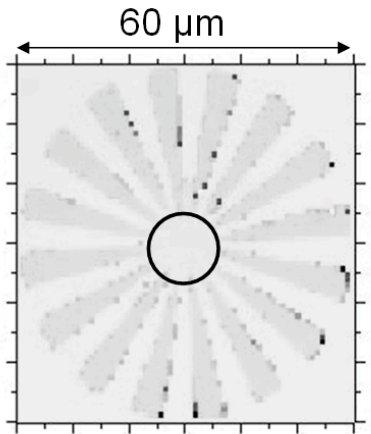

b) $20 \times$ objective

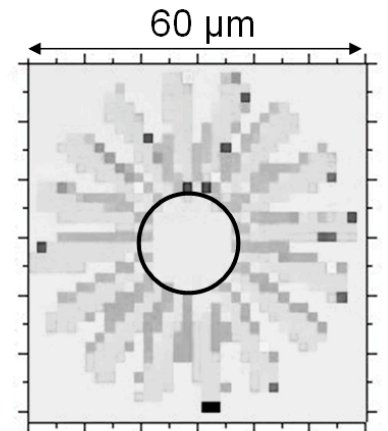

c) $10 \times$ objective

Figure 4: Measurement of ambiguous region with different objectives

Using the ambiguous regions diameter, the resolution has been calculated according to the method described above and the results are shown in table 2.

Table 2: Experimental calculated resolutions

\begin{tabular}{cccc}
$\begin{array}{c}\text { Number of } \\
\text { star }\end{array}$ & $\begin{array}{c}\text { Resolution } 10 \mathrm{X} \text { objective } \\
\text { in } \mu \mathrm{m}\end{array}$ & $\begin{array}{c}\text { Resolution } 20 \mathrm{X} \\
\text { objective in } \mu \mathrm{m}\end{array}$ & $\begin{array}{c}\text { Resolution } 50 \mathrm{X} \\
\text { objective in } \mu \mathrm{m}\end{array}$ \\
\hline 1 & 1.94 & 0.98 & 0.55 \\
2 & 2.04 & 0.96 & 0.55 \\
3 & 1.82 & 1.04 & 0.47 \\
4 & 1.91 & 1.04 & 0.47 \\
5 & 1.88 & 1.09 & 0.54
\end{tabular}

Although the applied evaluating system does not have rigid and objective criteria to find out the dimameter, the calculated values are stable for each objective and the variations of the obtained resolution within different stars are reasonable. This shows the high quality of the fabrication of stars. All the calculated results are larger than the specified values, which also show the need for such a practice oriented method in order to get information about the resolution performance of different instruments. Due to the diffraction limited resolution, results of $50 \mathrm{X}$ objective show the largest percentage differences in comparison to the values which are specified by the manufacturer. From these results, it is also clear that, diffraction limited resolution plays a decisive role especially at high magnifying objectives and it is not enough to give the number of pixel per field of view.

\subsection{Experiments with AFM}

Experiments are performed in the non-contact mode with a measurement range of $80 \mu \mathrm{m} \times 80 \mu \mathrm{m}$ while capturing 512 points per line. The Cantilever which is used has a diameter smaller than $8 \mathrm{~nm}$. As seen in figure 5 , the details of the ambiguous region are resolved, which could not be possible with other systems. In other words, the limits of manufacturing technology of this star are seen. After having 
calculated this region, it is found to be $0.45 \mu \mathrm{m}$. That means, resolution capacity of instruments whose resolutions are higher than this value could not be properly investigated.

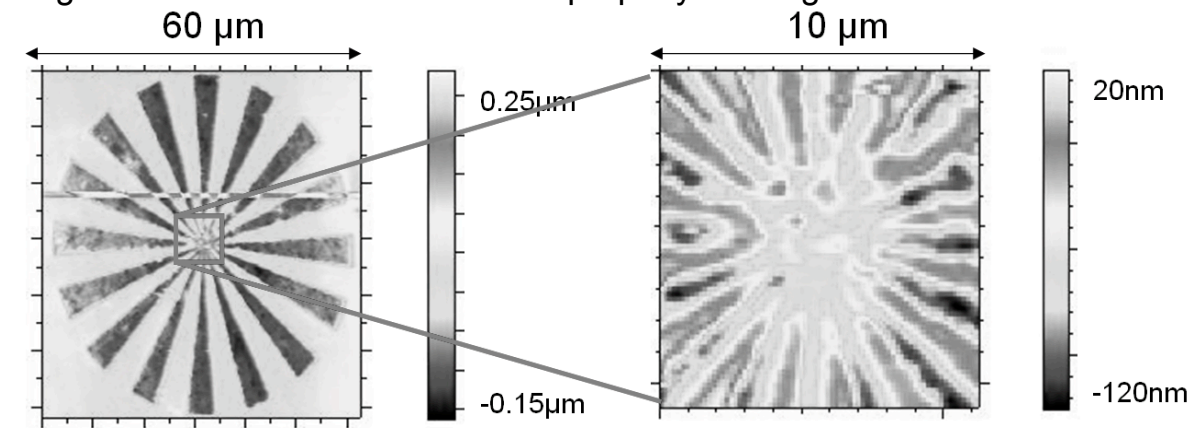

Figure 5: Measurement of Siemens-Stern with AFM

\subsection{Experiments with $\mathrm{CWL}$}

In comparison to other instruments, CWL has a low lateral resolution. Because of this reason, a Siemens star with a larger diameter $(100 \mu \mathrm{m})$ has been used. As a comparison, the same star has been also investigated with AFM. Due to the limited range of AFM $(80 \mu \mathrm{m} \times 80 \mu \mathrm{m})$, the whole star could not be measured. But the ambiguous region has been measured without any difficulties.

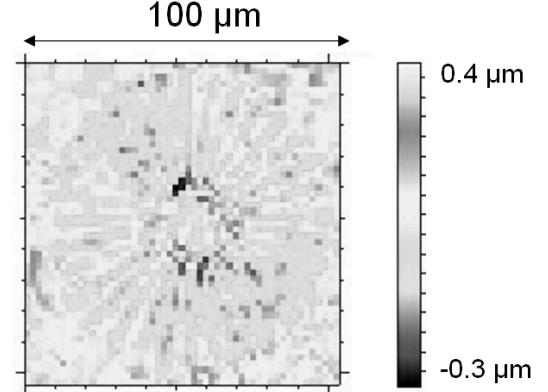

a) CWL

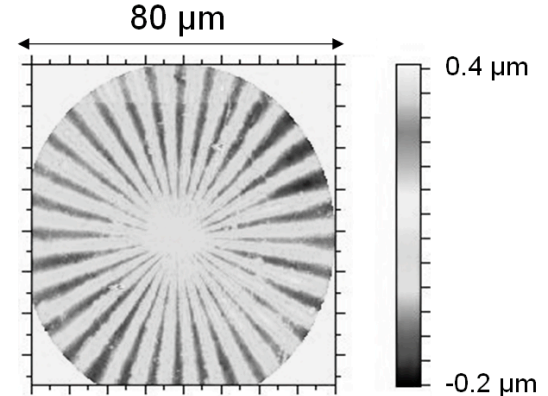

b) AFM

Figure 6: Measurement of the same Siemens-Star with a) CWL and b) AFM

Although the star has a lager diameter $(100 \mu \mathrm{m})$, structures of the CWL measurement could not be seen clearly. On the other hand, structures are clearly seen with AFM, which shows the reliability of the manufacturing method. In order to find out the possible reasons for the explanation of unclear images of $\mathrm{CWL}$, further investigations need to be fulfilled. Ignoring the unclear areas and using the height information from CWL measurements, the resolution is calculated about $3.5 \mu \mathrm{m}$. But this is the lower limit, because the disregarded aliasing effect is another important factor for the real resolution capacity.

\section{Discussions}

The concept of Siemens-Star, which is well-known in the field of image processing, has been applied to macro applications. Two different parts of experiments have been performed. In the first one, a comparison of resolution specifications given by manufacturer with experimental obtained resolutions has been done. Results show that, real resolution capacity of instruments is typically worse than theoretically calculated values. In the second part, not only the manufacturing limitation of such structures has been found out by AFM measurements, but also the problems encountered by the application of this technique to CWL have been shown. Other important information from AFM measurements is that, the ambiguous region in WLI measurements is not restricted by the manufacturing limitations but by the resolution performance of the instrument. It is also important to notice that, due to 32 separate branches the manufacturing tolerance is averaged by the number of the branches. For further investigations, a quite wide range of resolution values can be investigated with the same structure.

Acknowledgments:

The research and development project was funded by German Research Foundation (DFG) and has been carried out by the program of emphasis 1159 "New strategies for measurement and test techniques for production of microsystems and nanostructures". Project: "Mikro-Nano Messunsicherheit" (We 918/273). 


\section{$6 \quad$ Literature}

[1] Weckenmann, A.; Heidemann, L.; Tan, Ö.; Wiedenhoefer, Th.; Multisensor metrology for nanostructured technical surfaces; XII. Internationales Oberflächenkolloquium;2008; Chemnitz; P. $218-227$

[2] DIN $1319-1: 1995-01$, Basic Concepts in metrology

[3] Loebich, C.; Wueller, D.; Klingen, B.; Jaeger,A.; Digital Camera Resolution Measurement Using Sinusoidal Siemens Stars, SPIE, Vol. 6502, 65020N (2007); DOI:10.1117/12.703817

[4] Früke, R.; 2005 Würzburg, Entwicklung eines Rastermikroskopes für den Einsatz an Laborquellen im EUV Spektralbereich, Doktorarbeit, Bayerischen Julius-Maximilians-Universität Würzburg 